\title{
Transmisión transgeneracional del trauma psicosocial en comunidades indígenas de Argentina: percepción del daño en el pasado y presente y acciones autoreparatorias Transmissão transgeracional do trauma psicossocial em
comunidades indígenas na Argentina: a percepção de
dano no passado, presente e ações autorreparatórias
} Emilio losa', Tomás losa², Marcela Lucchese ${ }^{3}$, María Soledad Burrone ${ }^{4}$, Rubén Alvarado ${ }^{5}$, Eliecer Valencia ${ }^{6}$, Ruth Fernández ${ }^{7}$

\begin{abstract}
Resumen
El presente artículo indagó desde la perspectiva de los sujetos, con respecto a la transmisión transgeneracional del trauma psicosocial sufrido por comunidades Wichí del Norte Argentino. Se propuso identificar las experiencias percibidas como traumáticas por las comunidades Wichí, tanto pasadas como presentes, describiendo los relatos respecto a la vivencia del trauma y a las formas de socialización generadas en dicha comunidad. Esto tuvo presente las situaciones o acciones llevadas adelante en los últimos años, que pudieran ser consideradas como autoreparatorias. Se trata de un trabajo exploratorio de tipo cualitativo, realizado en comunidades Wichí de la Provincia de Salta, durante los años 2009, 2010 , y 2011. Fueron realizadas 30 entrevistas a caciques, mujeres, dirigentes y personas de las comunidades Wichí. Entre los resultados se reconoce la permanencia del daño en los grupos originarios y los mecanismos utilizados tanto por el Estado como por la sociedad para mantener esta situación, también se advierte un comportamiento de aislamiento y evasión ante el trauma, además se identifican secuelas y la manera en que éstas reproducen la situación de trauma. Fue también observado en la comunidad Wichí mecanismos de autoreparación en torno a la situación de trauma. La experiencia específica en relación con el pueblo Wichí reafirma los mecanismos de la transmisión transgeneracional del trauma psicosocial, surgiendo nuevas formas de organización de manera espontánea que develan el camino hacia la superación del trauma.
\end{abstract}

Palabras clave: salud indígena; población indígena; trastornos por estrés postraumático.

Trabajo realizado en Fundación Deuda Interna y Escuela de Salud Pública - Provincia de Córdoba, Argentina. 'Médico; Magister en Salud Pública; Presidente de la Fundación Deuda Interna - Provincia de Córdoba, Argentina.

¿Licenciado en Filosofía; Becario en el Consejo Nacional de Investigaciones Científicas y Técnicas (CONICET); Miembro Comité Honorario del Hospital Comunitario Dr. Salvador Mazza, Fundación Deuda Interna - Provincia de Córdoba, Argentina.

${ }^{3}$ Licenciada en Educación; Magister en Didáctica y Doctoranda en Ciencias de la Salud en la Escuela de Salud Pública, Facultad de Ciencias Médicas, Universidad Nacional de Córdoba - Provincia de Córdoba, Argentina.

${ }^{4}$ Médica; Magister en Salud Pública en la Escuela de Salud Pública, Facultad de Ciencias Médicas, Universidad Nacional de Córdoba - Provincia de Córdoba, Argentina. ${ }^{5}$ Doctor en Medicina; Especialista en Psiquiatría por la Escuela de Salud Pública, Facultad de Medicina, Universidad de Chile - Santiago, Chile; Miembro Comité Honorario del Hospital Comunitario Dr. Salvador Mazza, Fundación Deuda Interna - Provincia de Córdoba, Argentina.

${ }_{6}^{6}$ Profesor, Departamento de Epidemiología, Escuela de Salud Pública, Universidad de Columbia - New York (NY), USA. Profesor Adjunto en la Escuela de Salud Pública, Universidad de Chile - Santiago, Chile.

`Doctora en Medicina y Cirugía, Magister en Docencia; Profesora Titular en la Escuela de Salud Pública, Facultad de Ciencias Médicas, Universidad Nacional de Córdoba; Miembro Comité Honorario del Hospital Comunitario Dr. Salvador Mazza, Fundación Deuda Interna - Provincia de Córdoba, Argentina.

Dirección para correspondencia: Emilio losa - Fundación Deuda Interna - Avenida Cavallani, s/n - CP: 5189 - José de la Quintana - Provincia de Córdoba, Argentina-E-mail: emilioiosa@gmail.com

Soporto financiero: ningun.

Conflicto de interés: nada a declarar. 


\begin{abstract}
Resumo
O presente artigo investigou, a partir da perspectiva do sujeito, a transmissão transgeracional do trauma psicossocial sofrido pelas comunidades Wichí no Norte Argentino. Pretendeu-se identificar as experiências percebidas como traumáticas pelas comunidades Wichí, tanto no passado como no presente, descrevendo os relatos sobre a experiência do trauma e os modos de socialização gerados em tal comunidade. Foram levadas em conta as situações ou ações realizadas nos últimos anos, que poderiam ser consideradas como autorreparatórias. Tratou-se de um trabalho qualitativo exploratório, realizado nas comunidades Wichí da Província de Salta, durante 2009, 2010 e 2011. Foram realizadas 30 entrevistas com chefes, mulheres, líderes e pessoas das comunidades Wichí. Entre os resultados, é possível reconhecer a influência dos danos nos grupos originais e os mecanismos utilizados pelo Estado e pela sociedade para manter esta situação. Também destacam-se o comportamento de isolamento e a fuga perante o trauma. Foram identificadas as sequelas e a maneira que tais pessoas reproduzem a situação de trauma. Também mostraram-se, na comunidade Wichí, os mecanismos de autorreparação sobre a situação de trauma. A experiência específica com relação ao povo Wichí reafirma os mecanismos da transmissão transgeracional do trauma psicossocial, surgindo novas formas de organização emergente espontaneamente que revelam o caminho para superar o trauma.

Palavras-chave: saúde indígena; população indígena; transtornos de estresse pós-traumáticos.
\end{abstract}

\section{INTRODUCCIÓN}

La transmisión transgeneracional del trauma psicosocial se aborda asociada a los efectos de la guerra, a los problemas psíquicos en hijos de los sobrevivientes de campos de concentración o cuyos padres sufrieron otras experiencias traumáticas extremas ${ }^{1-4}$.

Se define un hecho traumático como la experiencia humana extrema que constituye una amenaza grave para la integridad física o psicológica de una persona y ante la cual la persona ha respondido con temor, desesperanza u horror intensos 5 . Tras haber experimentado, presenciado o escuchado un hecho de este tipo, pueden aparecer una serie de síntomas que escapa al control del superviviente y que le crea un profundo sufrimiento psicológico ${ }^{6,7}$. El estudio del trauma psicológico se relaciona con un hecho específico vivido por un individuo y está íntimamente vinculado con el desarrollo de acontecimientos sociales, políticos, y culturales ${ }^{8}$. En tal caso y si estas características se trasmiten a través de factores que condicionan la salud mental de las generaciones siguientes, se conforma el trauma transgeneracional ${ }^{9}$. En este contexto, se sostiene que la segunda y posiblemente la tercera generaciones tendrán que luchar con problemas psíquicos en tres aspectos: el equilibrio emocional, la autonomía, y el proceso identitario ${ }^{10-12}$. Por lo tanto, en la presente investigación, se abordó la transmisión transgeneracional del posible trauma psicosocial en las comunidades indígenas Wichí del norte argentino, víctimas de la llamada 'conquista del Chaco'.

Los Wichí (grupo indígena que habita territorios comprendidos en las actuales provincias del Chaco, Salta y Formosa, en la República Argentina y Sur de Bolivia y Paraguay) son alrededor del 40.000 a 50.000 personas $^{13}$. El estado Argentino a finales del siglo 19 y principios del 20 llevó adelante la ocupación militar del Chaco Central y Austral, provocando la muerte de millares de indígenas que habitaban la zona, sin existir datos fehacientes.
Después de 90 años, se comenzó un proceso de reparación histórica, entre las que se destaca: la firma del "Primer Manifiesto Wichí" (2009), la conformación de la "Comisión Nacional de Investigación del Genocidio para el Resarcimiento Histórico de los Pueblos Originarios de Argentina" y la organización del "Primer Congreso Nacional para la Unión de los Pueblos Originarios de Argentina”, donde se realizó la firma del Documento del Tratado Interétnico para la Refundación de la República Argentina (TIERRA), el 25 de mayo de 2010.

En este marco, el objetivo específico de la investigación planteó: identificar las experiencias traumáticas de las comunidades Wichí, describir la experiencia que los sujetos de las comunidades Wichí relatan respecto de la vivencia del trauma y de las formas de socialización generadas en dicha comunidad, e identificar las acciones comunitarias de carácter autoreparatorio.

\section{METODOLOGÍA}

Estudio exploratorio, con abordaje cualitativo sobre una muestra intencional. Se basó en palabras, historias y narraciones, cuyo interés es la dimensión subjetiva. Estudió los fenómenos en su medio natural, evaluando un sentido en función de los significados que los sujetos le atribuyen al mismo, de esta manera, se reconoce que existe una relación dinámica entre el mundo real y el sujeto. Se propuso analizar el significado de los sujetos a hechos, relaciones y prácticas, con la intención de construir conocimiento desde la singularidad de la situación. Aplica el método inductivo y la triangulación metodológica ${ }^{14}$.

Se llevó a cabo en diferentes comunidades Wichí de tres Departamentos de la Provincia de Salta, en Argentina. Se efectuaron 30 entrevistas ( 23 hombres y 7 mujeres, entre 30 y 70 años de edad). Los grupos entrevistados corresponden 
a: caciques, mujeres de caciques, dirigentes de organizaciones territoriales y personas de las comunidades Wichí. Para la selección de los sujetos, se consideró la disposición de las personas para contar la historia vivida por su comunidad y la participación en la lucha indígena.

El trabajo se organizó en etapas. En la primera, se sensibilizó a la comunidad. En una segunda, el equipo de investigación realizó las entrevistas y registró a través de cámaras y de grabaciones las experiencias vividas por los sujetos.

Los aspectos que orientaron las entrevistas fueron: la percepción del daño sufrido directamente o a terceros, los recuerdos e historias relacionadas al terrorismo de estado comentadas por los "mayores" (que son las personas de más edad y son los referentes de la comunidad desde el punto de vista político y espiritual), la relación inter-generacional respecto de estas vivencias, la situación actual del conflicto con el Estado, la percepción de perspectivas de solución al conflicto territorial y las nuevas formas de organización de la comunidad ${ }^{15}$.

A medida que se efectuaron las entrevistas, las primeras lecturas y el análisis comenzaron, se observaron recurrencias, diferencias y temáticas emergentes, se procedió a la codificación de la información. De manera conjunta a la codificación, fueron hechas lecturas de referentes teóricos y de la interpretación de este conjunto de situaciones y se construyeron categorías en torno a la problemática de la transmisión transgeneracional del trauma psicosocial.

\section{Consideraciones éticas}

El trabajo fue aprobado por el comité local, incluyendo las medidas necesarias para garantizar la protección de los sujetos, resguardando la participación voluntaria de todos los integrantes de la comunidad, y explicando en forma verbal a los entrevistados acerca del estudio que involucra la investigación, los objetivos del mismo, los procedimientos a seguir, los logros que se esperan del estudio, y la garantía de confidencialidad de su identidad.

\section{RESULTADOS}

A partir del análisis de las entrevistas, se reconocen las siguientes categorías:

- la permanencia del daño en los grupos originarios que es un proceso que devela los mecanismos utilizados por el Estado y la sociedad y especifica los contenidos que se trasmiten de generación en generación;

- silenciamiento y evasión son una posición de encapsulamiento ante el evento traumático;

- secuelas del evento del trauma vivido y re-traumatización;
- una relación cuestionada del estado-sociedad y comunidad Wichí; y

- el surgimiento de mecanismos de autoreparación.

En cuanto a la permanencia del daño en los grupos originarios se hace referencia a la forma en que se gesta el trauma a través de acciones del Estado, que se transmite a través de las relaciones familiares y que impactan en las trayectorias de vida de las siguientes generaciones. Particularmente, en este grupo, se advierten como mecanismos de construcción de la permanencia del daño: la situación de persecución, matanza, marginalidad, pobreza, e impunidad de las acciones que Estado y Sociedad sostienen y reproducen en relación a este grupo. Esto trae como consecuencia el no reconocimiento de sus costumbres, pertenencias e historia. En este sentido, también se observa que los mecanismos identificados se manifiestan, a través del tiempo, en un entramado de acciones que dan cuenta de su continuidad y de la herencia traumática circulando constantemente en la comunidad.

Se registraron las siguientes expresiones:

[...] lo siento mucho con las lágrimas cada comunidad que voy cuando empieza a hablar de la historia de las matanzas de lo que es un terror, se caen las lágrimas porque ellos le tienen esa herida que nadie les cura todavía. (Entrevista 15)

[...] ya no sufrimos de las matanzas pero estamos sufriendo pobreza [...] al no tener una casa digna vienen muchas enfermedades [....]. (Entrevista 13)

Estas cosas esas almas están como prisioneros todavía, porque son olvidados esas almas, que no podemos vivir de esta manera [...]. (Entrevista 2)

Acá hay marginación, esa marginación a veces duele [...]. (Entrevista 7)

Quien se acuerdan de nosotros que somos los indígenas sino que las cosas siguen que nosotros seamos cada día más pobre [...], seamos gente que no vale, no tengamos ley y seamos olvidados. (Entrevista 4)

[...] el exterminio más se hizo con los cabecillas, hoy queda la gente que se adaptó a ese modo de vida dominado por el criollo, creo que es parte de esa apatía, ese miedo que tiene el aborigen de juntarse con el criollo le cuesta mucho hablar, le cuesta mucho pedir, llevar sus inquietudes porque tiene cierto miedo a decir lo que le falta, lo que siente [...]. (Entrevista 16) 
No nos matan con armas pero nos matan por otro sistema ya. Nos están marginando todavía, no se cambió nada. (Entrevista 22)

En cuanto a los contenidos, se comunican experiencias de destrucción, pérdida y masacre vividas por los abuelos.

yo tengo este mensaje para todo el mundo, tengo este mensaje de una tristeza barbaridad [...] que yo he recorrido en mi chaco, he visto el masacre, he visto el cadáver, los gentes caídos como animales que tienen sus huesos sobre la faz de la tierra. (Entrevista 13)

El ejército asesinaba porque según dicen nuestros abuelos, había mucha paisanada, los mataron a todos, cuando había una mujer embarazada le partían la panza y le tiraban el chico afuera, que ya a punto de nacer llorando, el chiquito y ahí se cagan de risa, burla hace herejía. (Entrevista 6)

Respecto a la segunda categoría, el silenciamiento remite a establecer un manto de reserva sobre la experiencia traumática vivida y la evasión, se plantea en términos de ausencia y abandono del ambiente natural para alejarse y distanciarse de la situación de trauma. Así, la comunidad Wichí operó en una posición de encapsulamiento ${ }^{9}$ en referencia a las generaciones siguientes, no permitiendo hablar de los sucesos acontecidos. En relación al silenciamiento y evasión se advierte la manifestación de diferentes sensaciones como tristeza, dolor, miedo, y temores.

[...] los abuelos a veces no quieren hablar de esos temas de antes, de las matanzas, tienen miedo y no quieren contar, a todos nos da tristeza. (Entrevista 28)

A veces los abuelos cuentan, a veces no, pero si esta la tristeza [...]. (Entrevista 1)

Ellos se escapaban para cuidarse, no querían problemas con nadie y se metían más y más en el monte [...]. (Entrevista 18)

[...] me quedo muy triste de ver mis hermanos que están metidos en el corazón del monte como si fuera que ellos no tienen ningún funcionario, como si fuera que ellos no tienen el Estado, como si fuera que la Argentina no existe. (Entrevista 11)

[...] siguen siendo marginados olvidados con miedo de que algún rato le puede hacer lo mismo que en aquellos años todavía tienen esos temores, están lastimados sus mentalidades. (Entrevista 14)

[...] los que podían se escapaban, para el Sur se ha escapado muchísima gente aborigen, es una cosa triste, los han corrido a caballo, la gente se ha encajonado en una caída de agua, ahí lo han atrapado y han hecho iniquidades, mujeres embarazadas lo agarraban viva le metían un cuchillo. (Entrevista 30)

[...] se rebuscaban en el monte nomás, y buscaban para sostén de la vida y ellos no tenían coraje de enfrentar, pero más tenían miedo de las masacres y entonces trataban de escaparse para poder sobrevivirse. (Entrevista 29)

[...] con el tiempo se va convirtiendo un poco en un tema medio tabú pero en la memoria de la gente está, está ese recelo, al aborigen le cuesta integrarse porque por ahí no hay políticas para que pueda integrarse. (Entrevista 20)

Secuelas del evento del trauma vivido y re-traumatización. Tal como señala la bibliografía ${ }^{16}$, el trauma produce impacto individual y colectivo. Respecto a las consecuencias individuales, se generó daño en la totalidad biopsicosocial, que responde al trauma en sí mismo, a las condiciones psicobiológicas previas, a la pertenencia social, a la cosmovisión individual, y al grado de desarrollo de la conciencia de cada sujeto ${ }^{16}$. Por tanto, la consecuencia del daño del evento traumático es singular y única para cada persona.

[...] hasta el día de hoy estamos odiados [...] la escuela no tiene luz, no hay nada, no hay nada. Solo hambre. (Entrevista 8)

[...] me siento por un parte entristecido, y mucha pena que esos abuelos, que se van y les perdemos para siempre [...]. (Entrevista 5)

Con respecto a las consecuencias del daño colectivo, se conserva entre uno y otro sujeto una profunda relación de sentido y significación, y comparte ciertas características más generales, en las que se expresa su historicidad, es decir, su condición de experiencias humanas diversas que se igualan en su causalidad ${ }^{17}$.

Nosotros queremos progresar pero no nos dejan [...]. (Entrevista 12) 
Todos queremos disfrutar mientras vivimos, y lo que queda para nuestros hijos porque nosotros nos vamos a otro mundo, la gente se adueñó la tierra, toda la riqueza, no sé si duele a ustedes pero igualmente [...] ellos agarran toda esa riqueza y por ahí me da changuita y ellos me entregan lo que era mi plata. (Entrevista 18)

[...] me siento mucha preocupación de mis comunidades. (Entrevista 25)

El trauma sufrido fue tan severo que muchas personas señalan que marcó un quiebre vital y repercutió gravemente en su núcleo familiar.

[...] Hay jóvenes que van lejos, aquí no hay nada. Hasta día de hoy no hay nada. (Entrevista 22)

[...] El estado era el que ordenaba estas matanzas y yo no sé si el estado va a tratar de solucionar este problema porque yo no quiero que lleguemos a un enfrentamiento no deseado. (Entrevista 5)

Se observa en los relatos dificultad para lograr reinserción social y reconstruir un proyecto de vida, consecuencias a largo plazo del daño colectivo y persistencia del daño biopsicosocial. Esto se acentúa por el fenómeno de re-traumatización, y se evidencia que el trauma sigue vigente y toma distintas formas, pero sigue produciéndose en las mismas personas.

Vienen a comprar tierras y después los paisanos ¿a dónde van a quedar? Y el gobierno meta vender las tierras, y mientras tanto los paisanos ya se van creciendo, tiene hijos, se hacen muchos y después con poquita tierra no podemos hacer nada [...]. (Entrevista 9)

Nos han quitado las tierras a costo de las vidas, cuando vienen gente que tengan plata y compran a la gente que nos gobiernan y ellos son los que sacan el jugo de nuestras tierras y nos dejan las cáscaras para nuestra gente. (Entrevista 3)

Muchas veces la víctima directa de la situación violenta percibe su condición con mucha culpa, y estar "culpable" puede ser percibida por las generaciones siguientes de manera inconsciente.

No estamos aprovechando nada lo que es de la tierra no podemos trabajar, ni hacer nuestra propia casa con estos palos horcones no podemos sacar porque están siendo privados [...] si lo sacamos ya tenemos una denuncia [...] si queremos hacer una vivienda precaria tenemos que entrar a la fuerza, siendo de nosotros nos tenemos que sentir ladrones. (Entrevista 4)

Una relación cuestionada: estado-sociedad y comunidad Wichí

Se hace hincapié en la relación establecida entre estado-sociedad y comunidad Wichí, construida históricamente y caracterizada por sometimiento, indiferencia, y desconocimiento. En la actualidad, se reconoce una actitud diferente de la comunidad en términos de lo propio, de cuestionar las prácticas reproducidas en la sociedad y de generar otra forma de instalarse en la misma.

[...] nos han aprovechado la ignorancia que hemos tenido, el descuido total, pacíficamente, sin defensa, entonces digo: ¿por qué han hecho estas cosas? (Entrevista 7)

[...] y están los billetes, en fin, entonces nuestros hijos creyendo que esa gente son de buen corazón, como personas que han sido héroes de trabajar para el bienestar de todo la planeta Argentina, pero ahora se damos cuenta que esa figura ha sido el asesinato más grande que había en nuestro planeta. (Entrevista 10)

No era un hombre que decía le vamos a trabajar con esta gente, para que nosotros también tengamos parte del patrimonio de este pueblo y le ayudemos entre todos para que seamos una nación libre [...]. (Entrevista 15)

[...] me siento mucha preocupación de mis comunidades y de eso estoy trabajando y buscando para que el injusticia, lo que ha sido el masacre. (Entrevista 26)

\section{El surgimiento de mecanismos de autoreparación}

La generación de estrategias ante el potencial trauma transgeneracional y los movimientos sociales que fueron gestados desde las comunidades, como mecanismo de resiliencia, remite a las expresiones, acciones, y decisiones asumidas por la comunidad Wichí como posibilidad de cambio y expresión de hechos acontecidos:

Quizás tengamos tiempo de reorganizarnos de nuevo y ver entre todos como le llevamos nuestra nación y refundar de nuevo, como trabajamos de nuevo para no dañar nuestras tierras para los que vienen y aun no han nacido, yo creo que es basta ya hemos dado mucho como quinientos años de aprovechos. (Entrevista 30) 
Si queremos vivir bien, yo creo que se tenemos que unirnos [...]. (Entrevista 29)

[...] hay que construir de nuevo, no queda otro, si nosotros no lo hacemos ningún país se va a ocupar de nosotros. (Entrevista 2)

[...] yo deseo que nuestros hijos tengan posibilidad de construir y que tengan esa misma posibilidad de los que se hablan ser argentinos [...]. (Entrevista 5)

Queremos justicia y que nos escuchen, ya basta ya, esa es la lucha. No queremos por capricho sino por derecho. (Entrevista 10)

Nosotros no participamos de la marcha del bicentenario a Buenos Aires. No estamos de acuerdo lo que nos hace el estado, como puede ser que yo voy a festejar los 200 años mientras que nosotros estamos muriendo, somos olvidados, mientras que no nos dan atención y no hay solución de ninguna lucha [...]. (Entrevista 14)

A partir de esta categoría, fue posible identificar el surgimiento de una dimensión política, que construida colectivamente, otorga un reposicionamiento identitario, generando un nuevo vínculo con el restante de la sociedad.

[...] esto es responsabilidad de todos, no es por ser aborigen, este se tenemos que mirar arriba y que lo que nos pasa, que futuro nos está esperando, por eso estamos así trabajando, no busquemos ni cargo ni votos ni que vamos a ganar plata, nada, sino que queremos la justicia justa [...], eso es lo que nosotros estamos luchando. (Entrevista 18)

[...] entonces esta Refundación de la Argentina para que hagamos valer a todos, hasta el último hermano que tengamos en nuestro país, seamos gente que gocemos nuestro propio plantea de la tierra, que tengamos derecho a trabajar en igualdad, que tengamos nuestras cosas para comer todos los días diariamente. (Entrevista 1)

\section{DISCUSIÓN}

La transmisión transgeneracional del trauma psicosocial y de acuerdo con diversos autores ${ }^{9,18,19}$, es posible afirmar que este concepto es usado para dar cuenta del daño producido tanto en los directamente afectados, como a quienes, por razones de lazos familiares, comunitarios, idiomáticos, políticos, ideológicos, forman parte del entorno vital que sucede a estos a través del tiempo.

En este marco, se reconoce la permanencia del daño a través de las sucesivas generaciones. Autores como Brinkmann ${ }^{20}$ e Instituciones como Centro de Salud Mental y Derechos Humanos (CINTRAS) ${ }^{16}$ coinciden con el resultado expresado, identificando que la violencia institucional y legitimada por parte del estado constituyó un elemento central del ejercicio de poder.

Un elemento común identificado en la literatura, y que aporta un aspecto relevante a tener en cuenta para comprender el fenómeno de la transmisión transgeneracional del trauma, es el silenciamiento de las víctimas de experiencias traumáticas ${ }^{21-24}$. Silencio y negación forman una dinámica que se retroalimenta mutuamente en el contexto represivo ${ }^{25}$. Los resultados obtenidos en este estudio identifican al silenciamiento como experiencia traumática de la comunidad Wichí.

Otro aspecto de importancia en este trabajo remite a las diferentes actitudes asumidas por la comunidad Wichí, como son el cuestionamiento a la relación estado y sociedad y el surgimiento del mecanismo de autoreparación. En función de esto y en consonancia con Stern ${ }^{26}$, al no encontrar cabida en la memoria histórica oficial preponderante, para los sobrevivientes de tortura la interpretación propia de la historia vivida puede convertirse en factor de identidad ${ }^{26,27}$. Es una subcultura que el historiador Steve Stern ha definido como "memoria emblemática", caracterizada, en algunos casos, por ser "una ruptura lacerante no resuelta" $y$, en otros, por "la experiencia de vivir una vida de lucha, que ponía a prueba la consecuencia y el compromiso con los valores positivos, frente a una realidad de represión y miedo"26. Asimismo, en la bibliografía se identificaron aspectos relacionados a posibilidades de reparación del daño transgeneracional en jóvenes pertenecientes a las siguientes generaciones ${ }^{16,28}$. En esta investigación, los aspectos definidos como "autoreparatorios" son observados en individuos de distintos grupos etarios y generacionales.

\section{CONCLUSIONES}

Las consecuencias de los fenómenos de violencia política, tanto en períodos dictatoriales como democráticos, afectan a las generaciones siguientes de pueblos originarios damnificados. En la experiencia específica contra el pueblo Wichí, se reafirman los mecanismos de la transmisión transgeneracional del trauma psicosocial, tales como el silenciamiento, encapsulamiento de la experiencia traumática, aislamiento, y evasión. Surgen nuevas formas de organización de manera espontánea que develan el camino hacia la superación del trauma, mediante la toma de conciencia del daño, de manera que pueda ser un estímulo a la resistencia cultural y al desarrollo de un sentido de identidad. 


\section{REFERÊNCIAS}

1. Frazier KN, West O C, San Juste S, Goodman R. Trauma transgeneracional y CSA: casos reconceptualizar la participación de los jóvenes sobrevivientes de abuso sexual infantil. J Couns Salud Mental. 2009;3(1):22-33.

2. Almeida MES. A força do legado transgeracional numa família. Psicol Teor Prat. 2008;10(2):215-30.

3. Barnett S, Buckroyd J, Windle K. Eating disorders from parent to child: Mothers' perceptions of transgenerational effect. Counsel Psychother Res. 2005;5(3):203-11.

4. Daud A, Skoglund E, Rydelius P. Children in families of torture victims: transgenerational transmission of parents' traumatic experiences to their children. Int J Social Welfare. 2005;14(1):23-32.

5. American Psychiatric Association. Diagnostic and statistical manual of mental disorders, fourth edition: DSM-IV. Washington: American Psychiatric Association; 1994.

6. Pérez Sales P. Trauma, culpa y duelo. Hacia una psicoterapia integradora. Bilbao: Desclée de Brouwer; 2007.

7. Becker D. Trauma, duelo e identidad: Una reflexión conceptual. In: Becker D, Aguilar MI, Morales G, editores. Trauma psicosocial y adolescentes latinoamericanos: Formas de acción grupal. Santiago: Ediciones Chile-América CESOC; 1994.

8. Galvez Vilarreal JM, Halabí IP, Contreras FV. El proceso de traumatización en personas afectadas por terrorismo de estado. Un desarrollo conceptual. Cuad Psicol Jur. 2004;(1):1-13.

9. Madariaga C. Daños transgeneracional en Chile. Apuntes para una conceptualización. Rev Reflexión. 2003;30.

10. Mahler M. El nacimiento psicológico del infante humano. Buenos Aires: Marimar; 1975.

11. Erikson E. Dimensions of a New Identity. New York: Norton; 1974.

12. Barocas H, Barocas C. Manifestations of concentration camp effects on the second generation. Am J Psych. 1973;103(7):810-21.

13. Encuesta Complementaria de Pueblos Indígenas. Instituto Nacional de Asuntos Indígenas (INAI): Ministerio de Desarrollo Social, Presidencia de la Nación; 2005.

14. Magalhaes Bosi ML, Mercado FJ. Investigación cualitativa en los servicios de salud. Buenos Aires: Lugar; 2007.

15. Cufré Marchetto L. Violencia Social y sujetividad. In: García Aguirre, editor. Violencia, Identidad y globalización. Cruzana: Instituto de Investigaciones Histórico Sociales, Universidad Vera; 2004. p. 11.

16. Lagos M, Brasil VV, Brinkmann B, Scapucio M, editores. Daños transgeneracional consecuencia de la represión política del ConoSur. Santiago: LOM Ediciones; 2009.
17. Madariaga C. Tortura y trauma psicosocial. Rev Reflexión. 2001;27:5-9.

18. Rowland-Klein D. The transmission of trauma across generations: Identification with parental trauma in children of Holocaust survivors. In: Cayherall DR, editor. Handbook the stress, trauma and family. New York: Brunner-Routledge; 2004. p. 117-38.

19. Scapusio M, Pache S, Ortíz MC, Ruiz M. Efectos transgeneracionales del daño psicosocial ocasionado por el terrorismo de Estado. In: Lagos M, Brasil VV, Brinkmann B, Scapucio M, editores. Daño transgeneracional: consecuencias de la represión política en el cono sur. Santiago: LOM Ediciones; 2009. p. 329-440.

20. Brinkmann B. Itinerario de la impunidad, Chile 1973-1999. Un desafío a la dignidad. Santiago: CINTRAS; 1999.

21. Comisión Nacional sobre Prisión Política y Tortura. Informe [Versión electrónica]. Chile: Comisión Nacional sobre Prisión Política y Tortura [cited 2012 Mar 10]. Available from http://www.comisiontortura.cl/ listado_informes.html

22. Danieli Y. International Handbook of Multigenerational Legacies of Trauma. New York: Plenum Press; 1998.

23. Brinkmann B. El impacto de la negación y el silencio en torno a la tortura. In: Instituto Peruano de Educación en Derechos Humanos y la Paz. Reflexiones sobre la tortura. Lima: Instituto Peruano de Educación em Derechos Humanos y La Paz; 2005.

24. Kordon D, Edelman L. Por-venires de la memoria. Buenos Aires: Madres Plaza de Mayo; 2006.

25. Faúndez X, Cornejo M. Aproximaciones al estudio de la Transmisión Transgeneracional del Trauma Psicosocial. Rev Psicol. 2010;19(2):31-54.

26. Stern S. De la memoria suelta a la memoria emblemática: Hacia el recordar y el olvidar como proceso histórico (Chile, 1973-1998). In: Garcés M, Milos P, Olguín M, Pinto J, Rojas MT, Urrutia M, editores. Memoria para un nuevo siglo. Chile, miradas a la segunda mitad del siglo XX. Santiago: LOM Ediciones; 2000.

27. Brinkmann B, Guzmán JM, Madariaga C, Sandoval M. Daño transgeneracional en descendientes de sobrevivientes de tortura. In: Lagos M, Brasil VV, Brinkmann B, Scapucio M, editores. Daño transgeneracional: consecuencias de la represión política en el cono sur. Santiago: LOM Ediciones; 2009. p. 15-146.

28. Becker D, Castillo MI. Procesos de traumatización extrema y posibilidades de reparación. Santiago: ILAS; 1990.

Recibido en: 14/10/2012 Aprobado en: 05/02/2013 